AN. MED INTERNA (Madrid) Vol. 18, N. ${ }^{\circ} 11$, pp. 578-581, 200

\section{Variaciones estacionales en la hospitalización y mortalidad por insuficiencia cardiaca crónica en Vigo}

\author{
J. MONTES SANTIAGO, G. REY GARCÍA*, A. MEDIERO DOMÍNGUEZ*, \\ L. GONZÁLEZ VÁZQUEZ, E. PÉREZ FERNÁNDEZ, V. DEL CAMPO PÉREZ**, R. \\ PÉREZ ÁLVAREZ
}

Servicio de Medicina Interna. *Servicio de Documentación Clínica. **Unidad de Medicina Preventiva. Hospital Meixoeiro. Vigo
SEASONAL VARIATION IN CHRONIC HEART FAILURE HOSPITALI ZATIONS AND MORTALITY IN VIGO. SPAIN

\section{RESUMEN}

Objetivos: Son conocidas las variaciones estacionales en la morbimortalidad de procesos como la cardiopatía isquémica, pero hay escasos datos referentes a la insuficiencia cardiaca crónica (ICC). Este estudio analizó las variaciones estacionales en la hospitalización y muerte por ICC en Vigo.

Métodos: El Servicio de Documentación Clínica del Hospital Meixoeiro (419 camas, 167.000 habitantes >14 años) facilitó datos clínicos y estadísticos de las altas con diagnóstico de ICC (Código CIE9: 428). Los datos se dividieron en 3 periodos cuatrimestrales: invernal (noviembrefebrero, años 1997-98, 98-99 y 99-00), primaveral (marzo-junio, años 97, 98 y 99) y veraniego (julio-octubre, años 97, 98 y 99). Estudiamos también tasas de hospitalización y mortalidad en Cardiología, Medicina Interna y Geriatría, que atendieron al $81 \%$ de todas las ICC ingresadas.

Resultados: Hubo 1472 altas con el diagnóstico de ICC (52\% varones, $83 \%>65$ años, estancia media global: 13,8 días). Existió aumento de hospitalizaciones invernales tanto globales $-4,9 \%$ o (invierno) vs 3,2\% (verano)-, como de servicios médicos totales $-9,3 \%$ (invierno) vs $6,2 \%$, (verano). Este aumento invernal también se observó en servicios específicos: Cardiología: $+4,9 \%$, Medicina Interna: $+6,5 \%$, Geriatría: $+3,2 \%$. No hubo variaciones estacionales en la letalidad o mortalidad atribuible a ICC. El diagnóstico de ICC conllevó un riesgo de muerte 4 veces superior para los pacientes con dicho diagnóstico (OR: 3,81; IC: 3,28-4,42).

Conclusiones: El diagnóstico de ICC conlleva un exceso de mortalidad intrahospitalaria. Hubo un significativo aumento de hospitalizaciones invernales por ICC. Aquellas medidas dirigidas al control de este creciente problema deberían tomar en cuenta estas observaciones.

PALABRAS CLAVE: Insuficiencia cardiaca crónica. Variaciones estacionales. Hospitalizaciones.

\begin{abstract}
Objectives: Circannual variation in morbi-mortality for ischemic heart disease is well-known but there are few data focusing on chronic heart failure ( $\mathrm{CHF}$ ). This report analyzes seasonal variations in $\mathrm{CHF}$ hospitalizations and mortality in Vigo, Northwest of Spain.

Methods: Data on hospital discharge reports with a diagnosis of CHF (3-digit ICD9 code 428) were obtained from the Clinical Documen tation Service at Hospital Meixoeiro (419 beds, population: 167.000 inhabitants >14 years old). Data were divided in three 4-months periods: winter (november-february, years: 1997-98, $98-99$ and 99-00), spring (mars-june, years: 97, 98 and 99) and summer (july-october, years: 97, 98 and 99). Hospitalization rates and mortality were also stu died at Cardiology, Internal Medicine and Geriatrics (attending to $81 \%$ of patients with $C H F$ ).

Results: A total of $1.472 \mathrm{CHF}$ hospitalizations were registered (52\% male, $83 \%>65$ years, mean inhospital stay: 13.8 days). Significatives winter increases were noted in global hospitalizations $-4.9 \%$ (winter) vs. $3.2 \%$ ( summer) and overall medical services admissions $-9.3 \%$ (winter) vs. $6.2 \%$ (summer). These increases also were observed at specific medi cal services (Cardiology: $+4.9 \%$, Internal Medicine: $+6.5 \%$, Geriatrics: $+3.2 \%)$. There was not seasonal differences in letality or attributable mortality for CHF. Death in patients with a diagnosis of CHF was 4 times more likely. (OR:3.81; 95\% CI: 3.28-4.42).

Conclusions: There are a striking increase in winter hospitaliza tions for CHF. This diagnosis is associated with an excess of inhospital mortality. Preventive and therapeutic measures taking in account this observation are warranted to reduce the burden of this growing problem.
\end{abstract}

KEY WORDS: Chronic heart failure. Seasonal variations. Hospitaliza tions.

Montes Santiago J, Rey García G, Mediero Domínguez A, González Vázquez L, Pérez Fernández E, del Campo Pérez V, Pérez Álvarez R. Variaciones estacionales en la hospitalización y mortalidad por insuficiencia cardiaca crónica en Vigo. An Med Interna (Madrid) 2001; 18. 578-581.

\section{INTRODUCCIÓN}

La insuficiencia cardiaca crónica (ICC) constituye un problema grave de salud pública por su creciente prevalencia, el elevado número de ingresos hospitalarios y su elevada morta- lidad (1-6). Esto se complica por el progresivo envejecimiento de la población, produciéndose el ingreso por ICC en pacientes $>65$ años en las 3/4 partes de los casos. Así mismo, el 5\% de pacientes $>65$ años ingresados en un hospital presentan dicha patología (7). Los ingresos hospitalarios anuales por

Trabajo aceptado: 31 de Mayo de 2001

Correspondencia: Julio Montes Santiago. Servicio de Medicina Interna. Hospital Meixoeiro. 36200 Vigo. E-mail: jmontes@unimeixo.cesga.es. 
ICC aumentaron en España en un 71\% entre los años 1980 y 1993 (73.422 ingresos en este último año y más de 34.000 muertes) y la tasa de hospitalización en un $47 \%$ (7). Además la ICC no sólo es la primera causa de ingreso hospitalario sino también la primera causa de reingreso. A pesar de los indudables avances farmacológicos, que se han traducido en un aluvión reciente de guías y revisiones de esta entidad (8-17), repetidos estudios han señalado la elevada probabilidad de aquellos pacientes ancianos que sobreviven a un ingreso por ICC de reingresar por el mismo motivo y que puede oscilar entre el $27-75 \%$ a los 3 y 6 meses del alta $(18,20)$.

Aunque se han descrito acusadas variaciones estacionales en las tasas de hospitalización y muerte de procesos tales como muerte súbita, accidentes cerebrovasculares o infarto de miocardio (21), hay sorprendentemente pocos trabajos publicados de este cambio estacional en la ICC en países occidentales. Los estudios que se han ocupado del tema encuentran mayor tasa de ingresos en los meses invernales y lo atribuyen a factores tales como la mayor ingesta de sodio, episodios arrítmicos agudos, un incremento en la contaminación atmosférica o una superior incidencia de infecciones respiratorias, especialmente la gripe, en los meses fríos $(22,23)$. Nuestro objetivo en este trabajo fue describir y comparar las variaciones estacionales de hospitalización y muerte por insuficiencia cardiaca crónica en la población rural y urbana atendida por nuestro hospital en Vigo.

\section{MATERIAL Y MÉTODOS}

El estudio se realizó en el Hospital Meixoeiro de Vigo (419 camas, población rural y urbana de referencia: 167.000 habitantes >14 años). Dispone de un Servicio de Hemodinámica y Cirugía Cardiaca (alojado este último provisionalmente en otro centro en el último año considerado). El Servicio de Documentación Clínica facilitó datos clínicos y estadísticos, así como el listado total de casos con diagnóstico al alta de Insuficiencia Cardiaca (Código 428 de la Clasificación Internacional de Enfermedades, $9^{\mathrm{a}}$ edición MC; CIE9) como diagnóstico principal, durante el periodo invernal (considerado entre el 01/11 al 28/2 de los años 97-98, 98-99, 99-00), periodo primaveral (del 01/03 al 30/06 de los años 97, 98 y 99) y periodo veraniego (del 01/07 al 31/10 de los años 97, 98 y 99). También se estudiaron las hospitalizaciones en los Servi- cios de Cardiología, Medicina Interna y Geriatría, que atendieron al $81 \%$ de todas ICC ingresadas en servicios médicos (el $90 \%$ de todas ICC ingresaron en estos servicios) y que actuaron como consultores en la práctica totalidad de casos de ICC ingresadas. La comparación de los resultados obtenidos en los distintos periodos se realizó por el método chi-cuadrado. Se consideró significativo un valor de $\mathrm{p}<0,01$. También se calcularon los riesgos relativos y los intervalos de confianza del $95 \%$ por el método de Mantel-Haenszel.

\section{RESULTADOS}

En la tabla I y figura 1 se detallan las hospitalizaciones dividas por periodos estacionales. Se registraron un total de 1.472 altas por ICC. Globalmente y sin diferencias estacionales se observó que el $52 \%$ de los pacientes eran varones, el $83 \%>65$ años (con el 57\% > 75 años), con una estancia media de 13,8 días. Hubo aumento significativo de las hospitalizaciones en los meses más fríos. Este incremento de las hospitalizaciones invernales respecto a las veraniegas fue también significativo -excepto en Geriatría- al estudiar específicamente los servicios médicos más directamente involucrados en la atención a estos pacientes: Cardiología: $+4,9 \%$, Medicina Interna: $+6,5 \%$ y Geriatría: $+3,2 \%$. Se observó una menor letalidad por ICC en primavera, pero no hubo diferencias entre la letalidad por ICC entre meses invernales y veraniegos

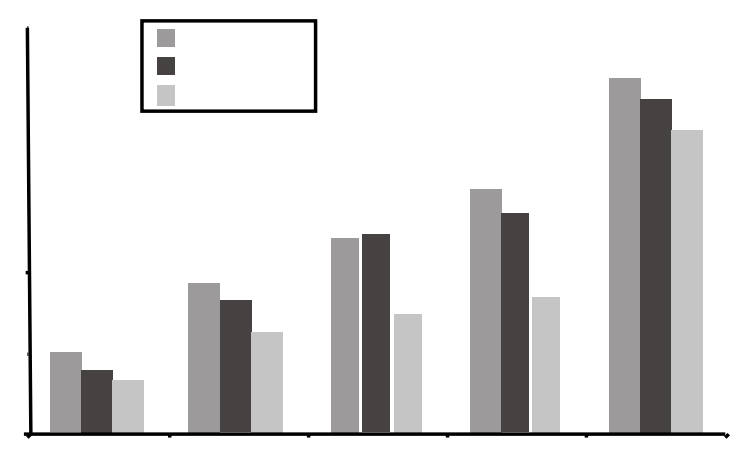

Fig. 1. Tasas de hospitalización estacional por insuficiencia cardiaca crónica. * $p<0,001$ respecto a verano.

TABLA I

DATOS EPIDEM IO LÓ GICOS Y CARACTERÍSTICAS ESTACIO NALES DE LA INSU FICIENCIA CARDIACA

\begin{tabular}{lrrr}
\hline & Invierno & Primavera & Verano \\
\hline No casos ICC & 605 & 505 & 362 \\
Varones (\%) & 50,4 & 52,6 & 54,4 \\
Estancia media (días) & 13,84 & 13,47 & 14,23 \\
Pacientes $>65$ años (>75 años) & $85 \%(59 \%)$ & $82 \%(56 \%)$ & $82 \%(58 \%)$ \\
Letalidad por ICC & $16,4 \%$ & $13,7 \%$ & $19,6 \%$ \\
(Exitus por ICC/ $n$ o casos ICC) & & & $12 \%$ \\
M ortalidad proporcional por ICC & $13 \%$ & & $11 \%$ \\
(Exitus por ICC/ $n$ o total exitus) & & & \\
\hline
\end{tabular}


ni en la mortalidad proporcional por ICC en los periodos considerados. El riesgo de muerte para pacientes con un diagnóstico de ICC fue casi 4 veces superior al de pacientes sin diagnóstico de ICC (Odds ratio: 3,81, IC 95\%: 3,28-4,42).

\section{DISCUSIÓN}

Encontramos en este estudio un acusado incremento invernal en las hospitalizaciones globales por ICC, que también se confirmó en los servicios médicos más directamente implicados en la atención a estos pacientes (aunque sin significación estadística en Geriatría). Los pacientes ingresados con el diagnóstico de ICC tuvieron además una mortalidad 4 veces mayor que los que no tuvieron ese diagnóstico, si bien no se observó mayor mortalidad invernal por ICC. Al igual que en otros estudios, el $82 \%$ de las hospitalizaciones por ICC se realizaron en pacientes $>65$ años, siendo en nuestro caso el $55 \%$ $>75$ años. De hecho, el 4,9\% del total de altas de pacientes $>65$ años de nuestro hospital en el periodo 1995-1999 (Montes J, datos no publicados) presentaron un diagnóstico de ICC, lo cual coincide también con otros estudios (7).

Varias han sido las hipótesis avanzadas para justificar el aumento de las hospitalizaciones y muerte por ICC durante los meses fríos. Se ha invocado, por ejemplo, que el clima frío provocaría una vasoconstricción periférica mediada simpáticamente que, mediante una elevación de la poscarga, podría precipitar un edema pulmonar, sobre todo en pacientes con patologías predisponentes como la hipertensión (23). No obstante, si dicha explicación fuese correcta debería observarse un exceso de hospitalizaciones y mortalidad por ICC en las provincias norteñas de España con respecto a las meridionales, cosa que no sucede en una publicación reciente sobre la variación geográfica de la mortalidad y hospitalizaciones en España por ICC, en el periodo 1980-1993 (24). Es destacable en este estudio la notable excepción de Ponteve- dra entre las provincias norteñas, en la que si bien no se registró aumento en las tasas de mortalidad por ICC, sí se observó incremento en la tasa de hospitalización desde 256326 por 100.000 habitantes $>45$ años (periodo 1983-1989) a 464-584 (periodo 1989-1993), situándose entre las más altas de España. Para el área de Pontevedra Sur, atendida por nuestro hospital, esta tendencia se mantiene para el periodo 199599. (Tasa de hospitalización por ICC: 574 por 100.000 habitantes >45 años, IC 95\%: 556-592; Montes J, datos no publicados).

Otra hipótesis liga los niveles de contaminación ambiental con el aumento de las hospitalizaciones y muertes por procesos cardiovasculares, incluyendo ICC $(25,26)$. Recientes estudios multicéntricos europeos (Proyecto APHEA) (27), norteamericanos (28) y españoles (Proyecto EMECAM) (29) han aportado evidencias de tal asociación. No obstante, hay que reseñar que en el último estudio citado, realizado en 14 grandes ciudades españolas, no se demostró, para el caso concreto de la ciudad de Vigo, una asociación entre contaminación y aumento de mortalidad cardiovascular (30).

Además de las medidas para reducir la contaminación atmosférica, que dependen en primera instancia de autoridades municipales y políticas, el otro gran factor precipitante de las hospitalizaciones por IC son las infecciones respiratorias, sobre todo la gripe, que están presentes en el 10-20\% de las hospitalizaciones por ICC $(31,32)$, llegando al $37 \%$ en alguna serie española (33). En este sentido, la vacunación antigripal sería una medida extradordinariamente coste-efectiva ya que, amén de la reducción de la tasa de reinfartos de miocardio (34), se ha comunicado que puede reducir las hospitalizaciones por ICC entre un $27-37 \%$, sobre todo en las personas ancianas (35-37).

Nuestros datos, de confirmarse en otras autonomías y a nivel nacional, pueden permitir a las autoridades sanitarias el diseño de estrategias preventivas adecuadas para un mejor manejo de esta moderna epidemia cardiovascular.

\section{Bibliografía}

1. Sáenz de la Calzada C. Insuficiencia cardiaca. Un problema de salud pública. An Med Interna (Madrid) 1999; 16: 551-2.

2. Cowie MR, Mosterd A, Wood DA, Deckerst JW, Poole-WilsonPA, Sutton GC, Grobbee DE. The epidemiology of heart failure. Eur Heart J 1997; 18: 208-23.

3. Hoes AW, Mosterd A, Grobbee DE. An epidemic of heart failure? Recent evidence from Europe. Eur Heart J 1998; (Suppl L): L2-L8.

4. Brotons C, Moral I, Ribera A, Pérez G, Bustins M, Permanyer-Miralda G. Tendencias en la morrbimortalidad por insuficiencia cardiaca en Cataluña. Rev Esp Cardiol 1998; 51: 972-6.

5. Sáez T, Suárez C, Blanco F, Gabriel R. Epidemiología de las enfermedades cardiovasculares en la población anciana española. Rev Esp Cardiol 1998; 51: 864-73.

6. Ferreira Montero IJ. Insuficiencia cardiaca: mayor morbilidad, menor mortalidad ¿vamos por el buen camino? Rev Esp Cardiol 2000: 53: 767-9.

7. Rodríguez Artalejo F, Guallar-Castillón P, Banegas Banegas JR, del Rey Calero J. Trends in hospitalization and mortality for heart failure in Spain, 1980-1993. Eur Heart J 1997; 18: 1771-9.

8. Celemín I, Sánchez Mata N, Rodríguez Entem F, De la Torre JM, Vázquez de Prada JM. Medicina basada en la evidencia. Tratamiento farmacológico de la insuficiencia cardiaca crónica. Monocardio 1999; 1: 206-12.
9. Acquatella H. Miocardiopatía dilatada: avances recientes y tratamiento actual. Rev Esp Cardiol 2000; (Supl. 1): 19-27.

10. Conthe Gutiérrez P, Pacho Jiménez E. El tratamiento de la insuficiencia cardiaca. Rev Clin Esp 2000; 200: 551-62.

11. Hess OM. State of the art: current treatment of heart failure. Eur Heart J 2000; (Suppl A): A13-A16.

12. Cleland JGF. Exitos y fracasos del tratamiento actual de la insuficiencia cardiaca. En: Grandes temas de la cardiología. American College of Cardiology. Barcelona: Medical Trends 2000; 69-80.

13. Lonn E, McKelvie R. Drug treatment in heart failure. BMJ 2000; 320: 1188-92.

14. Castro Beiras A, Rodríguez JA. Insuficiencia cardiaca. Presente y futuro del tratamiento. En: Enfermedades cardiovasculares. Nutricion, Genética y Epidemiología. De Oya M, Garcés C, eds. Madrid: Ed. Doyma, 2000; 133-48.

15. Heart Failure Society of America (HFSA). Guía de actuación para el tratamiento de los pacientes con insuficiencia cardiaca debida a disfunción sistólica ventricular izquierda. Enfoques farmacológicos. Congestive Heart Failure (ed. esp.) 2000; 1: 1-34.

16. Grupo de trabajo de ICC de la Sociedad Española de Medicina Interna. Insuficiencia cardiaca: recomendaciones diagnósticas y terapéuticas. Coordinador: Conthe P, ed. Madrid: ed. Jarpyo; 2000. 
17. Sociedad Española de Cardiología. Guías de práctica clínica en la insuficiencia cardiaca y shock cardiogénico. Sociedad Española de Cardiología. Madrid, 2000; tomo 1; 123-79.

18. Krumholz HM, Parent EM, Tu N, Vaccarino V, Wang Y, Radford MJ, et al. Readmission after hospitalization for congestive heart failure among Medicare beneficiaires. Arch Intern Med 1997; 157: 99-104.

19. Chin $\mathrm{MH}$, Goldman L. Correlates of early readmission or death in patientes with congestive heart failure. Am J Cardiol 1997; 79: 1160-4.

20. Montoto Otero C, Agudo de Blas P, Casado Mesegué E, et al. Reingreso hospitalario por insuficiencia cardiaca en un hospital general. An Med Interna 2000; 17: 71-4.

21. Spencer FA, Goldberg RJ, Becker RCGore JM. Seasonal distribution of acute myocardial infarction in the second national registry of myocardial infarction. J Am Coll Cardiol 1998; 31: 1226-33.

22. Morris RD, Naumova EN. Carbon monoxide and hospital admissions for congestive heart failure: evidence of an increased effect at low temperatures. Environ Health Perspect 1998; 106: 649-53.

23. Boulay F, Berthier F, Sisteron O, Gendreike Y, Gibelin P. Seasonal variation in chronic heart failure hospitalizations and mortality in France. Circulation 1999; 100: 280-6.

24. Rodríguez-Artalejo F, Guallar-Castillón P, Banegas Banegas JR, del Rey Calero J. Variación geográfica en las hospitalizaciones y en la mortalidad por insuficiencia cardíaca congestiva en España, 19801993. Rev Esp Cardiol 2000; 53: 776-82.

25. Schwartz J. Air pollution and hospital admissions for heart disease in eight US counties. Epidemiology 1999; 10: 17-22.

26. Burnett RT, Dales RE, Brook JR, Raizenne ME, Krewski D. Association between carbon monoxide levels and hospitalizations for congestive heart failure in the elderly in 10 Canadian cities. Epidemiology 1997; 8: 162-7.

27. Katsouyanni K, Toulomi G, Spix C, Schwartz J, Balducci F, Medina S, et al. Short-term effects of ambient sulphur dioxide and particulate mat- ter on mortality in 12 European cities: results from times series data from the APHEA project: Air Pollution and Health: A European Approach. BMJ 1997; 314: 1568-63.

28. Samet JM, Dominici F, Curriero F, Coursac I, Zeger SL. Fine particulate air pollution and mortality in $20 \mathrm{U}$. S. Cities, 1987-1994. N Engl J Med 2000; 343: 1742-9.

29. Sunyer J. El estudio EMECAM sobre los efectos de la contaminación atmosférica. Rev Esp Salud Publ 1999; 73, n². (marzo-abril 1999, edición online).

30. Taracido Trunk M, Figueiras A, Castro Lareo I. Efecto a corto plazo de la contaminación atmosférica sobre la mortalidad. Resultados del proyecto ENECAM en la ciudad de Vigo, 1991-1994. Rev Esp Salud Pública 1999; 73, nº 2. (marzo-abril 1999, edición online).

31. Chin $\mathrm{MH}$, Goldman L. Factors contributing to the hospitalization of patientes with congestive CHF. Am J Public Health 1997; 87: 643-8.

32. Bennett ST, Huster GA, Baker SL, Milgrom LB, Kirchgassner A, Birt $\mathrm{J}$, et al. Characterization of the precipitants of hospitalization for $\mathrm{CHF}$ decompesation. Am J Crit Care 1998; 7: 168-74.

33. Ramos Polledo V, Pita Fernández S, De la Iglesia Martínez F, Pellicer Vázquez C, Nicolás Miguel R, Diz-Lois Martínez F, et al. Etiología, características clínicas, causa desencadenante, tipo de disfunción ventricular, estancia media y mortalidad de 305 pacientes ingresados por insuficiencia cardiaca.. An Med Interna (Madrid) 2000; 17: 19-24.

34. Naghavi M, Barlas Z, Siadaty S, Naguib S, Madjid M, Casscells W. Association of influenza vaccination and reduced risk of recurrent myocardial infarction. Circulation 2000; 102: 3039-45.

35. Nichol KL, Margolis KL, Wuorenma J, Von Sternberg T. The efficacy and cost effectiviness of vaccination against influenza among elderly people living in the community. N Engl J Med 1994; 331: 778-84.

36. Kaiser L, Hayden FG. Hospitalizing influenza in adults. Curr Clin Top Infect Dis 1999; 19: 112-34.

37. Couch RB. Prevention and treatment of influenza. N Engl J Med 2000; 343: $1778-87$ 